\title{
Diabetes mellitus accelerates left ventricular diastolic dysfunction through activation of the renin-angiotensin system in hypertensive rats
}

\author{
Shigefumi Fukui ${ }^{1}$, Yoshihiro Fukumoto ${ }^{1}$, Jun Suzuki ${ }^{1}$, Kenya Saji ${ }^{1}$, Jun Nawata ${ }^{1}$, Tsuyoshi Shinozaki ${ }^{1}$, \\ Yutaka Kagaya $^{1}$, Jun Watanabe ${ }^{1}$ and Hiroaki Shimokawa ${ }^{1,2}$
}

Diabetes mellitus (DM) is a major risk factor for heart failure, independent of coronary artery disease or hypertension (HT). Therefore, our study was designed to examine the mechanisms of DM-induced left ventricular (LV) diastolic dysfunction. In this study, we made five different 10-week treatment groups of Dahl salt-sensitive rats as follows: Control; a low-salt $(0.5 \% \mathrm{NaCl})$ diet, $\mathrm{HT}$; a high-salt ( $5 \% \mathrm{NaCl}$ ) diet, DM; a low-salt diet with streptozotocin (STZ) injection ( $30 \mathrm{mg} \mathrm{kg}^{-1}$ i.p.), HT+DM; a highsalt diet with STZ injection, and the Olmesartan group; a high-salt diet with STZ treated with an angiotensin receptor blocker, olmesartan $\left(1 \mathrm{mg} \mathrm{kg}^{-1} \mathrm{day}^{-1}\right)$. Cardiac diastolic dysfunction with a preserved systolic function was noted in the HT group, and was most prominently noted in the HT+DM group, characterized by enhanced cardiac fibrosis, whereas the extent of $\mathrm{HT}$ and myocardial hypertrophy was comparable between the two groups. Myocardial expressions of collagen III, transforming growth factor-p2, angiotensin-converting enzyme (ACE), angiotensin II type-1 receptor and myocardial oxidative stress (evaluated by 4-hydroxy-2-nonenal-modified protein) were mostly enhanced in the HT+DM group. Importantly, there was a positive correlation between the extent of diastolic dysfunction and that of myocardial ACE expression. All these cardiac abnormalities induced by DM and HT were ameliorated in the olmesartan group. These results indicate that DM accelerates diastolic dysfunction in hypertensive heart disease through activation of the renin-angiotensin system, with subsequent inflammatory and oxidative stresses and myocardial fibrosis, suggesting that an inhibition of the system is effective for the treatment of diastolic dysfunction in this combined disorder.

Hypertension Research (2009) 32, 472-480; doi:10.1038/hr.2009.43; published online 24 April 2009

Keywords: diabetes mellitus; diastolic dysfunction; fibrosis; renin-angiotensin system

\section{INTRODUCTION}

Heart failure has been considered to be a complex clinical syndrome that can result from any structural or functional cardiac disorder that impairs the ability of the ventricle to fill with or eject blood. ${ }^{1}$ Recently, a number of studies have shown that heart failure could also occur in patients with a preserved left ventricular (LV) ejection fraction, which is often observed in patients with hypertensive heart disease. ${ }^{2}$ This phenomenon was termed as diastolic heart failure (DHF) in contrast to systolic heart failure. ${ }^{3}$ It is now widely accepted that DHF is also a major cardiovascular disorder with poor prognosis, accounting for approximately half of the patients with a heart failure symptom. ${ }^{4,5}$

It has been reported that nearly $30 \%$ of patients with DHF also have diabetes mellitus (DM). ${ }^{6,7} \mathrm{DM}$ is a major risk factor for heart failure, ${ }^{6,8}$ because the disorder causes functional, biochemical and morphological abnormalities of cardiomyocytes, independent of coronary artery disease or hypertension (HT). ${ }^{6}$ In the development of diabetic cardiomyopathy, DHF can be observed at an early stage, followed by progressive systolic heart failure at a later stage. ${ }^{9,10}$ It was suggested that patients with both DM and HT complicate LV diastolic dysfunction more severely than those with either condition alone. ${ }^{11}$ Earlier studies have shown that DM exacerbates LV remodeling and heart failure after myocardial infarction in mice, wherein angiotensin II plays an important pathogenetic role. ${ }^{12,13}$ Indeed, angiotensinconverting enzyme (ACE) inhibitors are beneficial for coronary blood flow and cardiac performance in hypertensive diabetic rats. ${ }^{14}$ However, the detailed mechanisms of DM-induced additive deleterious effects on LV diastolic dysfunction remain to be fully elucidated. Thus, this study was designed to examine whether the cardiac reninangiotensin system (RAS) plays an important role in the pathogenesis of DM-induced LV diastolic dysfunction in the presence of HT in rats.

\section{METHODS}

All procedures were carried out according to the protocols approved by the Institutional Committee for Use and Care of Laboratory Animals of Tohoku University. The authors had full access to the data and take full responsibility for its integrity. All authors have read and agreed to the article as it has been written.

${ }^{1}$ Department of Cardiovascular Medicine, Tohoku University Graduate School of Medicine, Sendai, Japan and ${ }^{2}$ Technology Agency, CREST, Tokyo, Japan Correspondence: Dr Y Fukumoto, Department of Cardiovascular Medicine, Tohoku University Graduate School of Medicine, 1-1 Seiryo-machi, Aoba-ku, Sendai 980-8574, Japan. E-mail: fukumoto@cardio.med.tohoku.ac.jp

Received 3 December 2008; revised 18 February 2009; accepted 6 March 2009; published online 24 April 2009 


\section{Animal preparation}

Male Dahl salt-sensitive rats (DIS/Eis, Japan SLC Inc., Shizuoka, Japan) were fed a normal diet from weaning until 7 weeks of age. Thereafter, they were randomly divided into the following five groups for 10-week treatments: a regular chow containing $0.5 \% \mathrm{NaCl}$ with or without streptozotocin (STZ) injection (DM and control groups, $n=12$ and 20, respectively), a high-salt (5\% $\mathrm{NaCl})$ diet for 10 weeks with or without STZ injection (HT+DM and HT groups, $n=12$ and 14 , respectively) and a high-salt diet with STZ injection and treated with olmesartan $\left(1 \mathrm{mg} \mathrm{kg}^{-1} \mathrm{day}^{-1}\right.$ in the drinking water) from 11 weeks of age (olmesartan group, $n=12$ ). They were given an i.p. injection of freshly prepared STZ $\left(30 \mathrm{mg} \mathrm{kg}^{-1}\right)$ in a $0.1 \mathrm{moll}^{-1}$ citrate buffer $(\mathrm{pH} 4.5)$ or citrate buffer alone at 11 weeks of age. Tail vein blood glucose samples were measured 3 days after injection of STZ or placebo to ensure the induction of diabetes. STZ-treated rats with nonfasted blood glucose level over $200 \mathrm{mg}$ per $100 \mathrm{ml}$ were determined to be diabetic. The rats with nonfasted blood glucose level below $200 \mathrm{mg}$ per $100 \mathrm{ml}$ received additional multiple i.p. injections of STZ in the same manner over a 2-week period, until the blood glucose level reached over $200 \mathrm{mg}$ per $100 \mathrm{ml}$. After the hemodynamic studies, venous blood samples were collected to ensure the continuation of high blood glucose levels. All animals were housed under controlled temperature and a 12-h light/dark cycle. The diet and tap water were provided ad libitum throughout the experiment. We determined the sodium amount of the high-salt diet as being $5 \% \mathrm{NaCl}$ and the duration of HT and diabetes as 10 and 6 weeks in this study, because our preliminary study indicated that STZ-treated rats fed a diet containing $8 \%$ $\mathrm{NaCl}$ had a high mortality rate during the study period for 8 weeks of diabetes, whereas STZ-treated rats with $5 \% \mathrm{NaCl}$ had diastolic dysfunction without death during the study period for 6 weeks of diabetes. The present dose of olmesartan $\left(1 \mathrm{mg} \mathrm{kg}^{-1} \mathrm{day}^{-1}\right.$, PO) was also determined as being a subdepressor dose on the basis of our preliminary study, in which 0.6 and $1 \mathrm{mg} \mathrm{kg}^{-1}$ day $^{-1}$ of olmesartan did not decrease tail-cuff systolic blood pressure measured at 2-week intervals in Dahl salt-sensitive rats. Its sub-depressor dose is relatively variable to be determined, because earlier studies using a Dahl saltsensitive rat model have reported that 0.6 or $3 \mathrm{mg} \mathrm{kg}^{-1} \mathrm{day}^{-1}$ of olmesartan did not lower blood pressure but that $2.5 \mathrm{mg} \mathrm{kg}^{-1}$ day $^{-1}$ of the drug did. ${ }^{15-17}$ Therefore, we have carefully carried out our preliminary study in our system and determined that 0.6 and $1 \mathrm{mg} \mathrm{kg}^{-1} \mathrm{day}^{-1}$ of olmesartan were sub-depressor doses. Systolic blood pressure and heart rate were measured every 2 weeks with a tail-cuff system (MK-2000, Muromachi, Tokyo, Japan). ${ }^{18,19}$

\section{Echocardiographic study}

Transthoracic M-mode and Doppler echocardiographic studies were carried out at 7 and 17 weeks of age, using an ultrasonographic system (Aplio, Toshiba, Tokyo, Japan) with a $10 \mathrm{MHz}$ transducer as described earlier. ${ }^{18-20}$ They were anesthetized with ketamine $\mathrm{HCl}\left(50 \mathrm{mg} \mathrm{kg}^{-1}\right.$, i.p. $)$ and xylazine $\mathrm{HCl}$ $\left(10 \mathrm{mg} \mathrm{kg}^{-1}\right.$, i.p.), and were held in the half left-lateral position under spontaneous respiration. M-mode echocardiograms were recorded with short-axis views at the papillary muscle level of the LV at an M-speed of $100 \mathrm{~mm} \mathrm{~s}^{-1}$. Thickness of the interventricular septum and of the LV posterior wall at end diastole (LVPWT) and LV diameters at end diastole (LVDd) and end systole (LVDs) were measured according to the American Society of Echocardiography leading edge method. ${ }^{21}$ End diastole and end systole were determined at the point of the most posterior and anterior excursions of LVPW, respectively. LV fractional shortening (LVFS) (\%) was calculated as: LVFS $=(($ LVDd - LVDs $) /$ LVDd $) \times 100$. Doppler transmitral inflow velocity was recorded from an apical 4-chamber view, with the sample volume adjusted to the smallest size available $(1.0 \mathrm{~mm})$. Peak early diastolic filling velocity (E velocity), peak filling velocity at atrial contraction (A velocity) and their ratio (E/A ratio) were measured for consecutive five cardiac cycles. ${ }^{22}$

\section{Hemodynamic study}

Hemodynamic studies were carried out using a $2 \mathrm{~F}$ high-fidelity manometertipped catheter (Millar Instruments, Houston, TX, USA) as described earlier. ${ }^{18-20}$ At 17 weeks of age, after the echocardiographic study, the catheter was retrogradely introduced through the right carotid artery into the LV under general anesthesia. Tracings of LV pressure and ECG were digitized and processed by a computer system, to determine LV end-diastolic pressure, the peak positive and negative values of its first derivatives $(\mathrm{dP} / \mathrm{dt})$ and an index of LV relaxation, $\tau$, using a nonzero asymptote method as described earlier. ${ }^{23}$ The LV end-diastolic pressure was determined from consecutive five cardiac cycles as the pressure at the point just before the onset of an increase in LV systolic pressure.

\section{Histological examination}

After the hemodynamic studies, the heart and the lungs were immediately harvested. The LV was carefully separated from the right ventricle, the atria and great vessels, and the weights of the LV and the lungs were measured. The ratio of LV weight to body weight was calculated as an index of LV hypertrophy. ${ }^{19,24}$ Apical portion of the LV below the papillary muscle was fixed with a phosphate-buffered $10 \%$ formalin solution for $24 \mathrm{~h}$, embedded in paraffin, sectioned at $1.5 \mu \mathrm{m}$ and stained with either hematoxylin and eosin or a sirius red stain to evaluate the extent of interstitial fibrosis. ${ }^{19,25,26}$ The remaining LV was immediately placed in liquid nitrogen and stored at $-80^{\circ} \mathrm{C}$ for western blot analysis and mRNA quantification. Cardiomyocyte cross-sectional diameter was measured in the cells that were cut transversely with both a visible nucleus and an unbroken cellular membrane. ${ }^{19}$ At least 30 cardiomyocytes were measured per heart, and the average was used for analysis. The percentage area of interstitial fibrosis in the LV at the papillary muscle level in the slides stained with the sirius red stain was determined as described earlier, ${ }^{18,19,25-28}$ using a commercially available computer software (Mac scope v 2.5, Mitani, Fukui, Japan).

\section{Western blot analysis}

Frozen LV tissues were homogenized in lysis buffer containing $50 \mathrm{~mm}$ Tris- $\mathrm{HCl}$ ( $\mathrm{pH}$ 7.4), $150 \mathrm{~mm} \mathrm{NaCl}, 5 \mathrm{~mm}$ EDTA, $10 \mathrm{~mm}$ sodium pyrophosphate, $10 \mathrm{~mm}$ $\beta$-glycerophosphate, $1 \mathrm{~mm}$ sodium orthovanadate, $10 \mathrm{~mm} \mathrm{NaF}, 1 \mathrm{~mm}$ ditbiothreitol, $1 \%$ Triton X-100, 10\% glycerol and a $1 \%$ protease inhibitor cocktail. The homogenate of the LV was centrifuged at 15000 r.p.m. for $20 \mathrm{~min}$. The protein concentration of the supernatant was determined with bovine serum albumin as a standard protein. The same amount (10-30 $\mu$ g for each experiment) of extracted protein was loaded for SDS-polyacrylamide gel electrophoresis and then transferred onto polyvinylidene difluoride membranes. ${ }^{19}$ The membranes were probed with an antibody to ACE (sc-20791, at 1:2000 dilution, from Santa Cruz Biotechnology Inc., Santa Cruz, CA, USA), to 4-hydroxy-2nonenal-modified protein (N213220, at 1:5000 dilution, from NOF Medical Department, Tokyo, Japan) and to glyceraldehydes-3-phosphate-dehydrogenase (GAPDH) (sc-20357, at 1:2000 dilution, from Santa Cruz Biotechnology Inc.). Signals were visualized by the ECL detection system (GE Healthcare UK Ltd, Buckinghamshire, UK). Subsequently, the amounts of ACE and 4-hydroxy-2nonenal expression were normalized to that of GAPDH expression.

\section{Real-time PCR}

Total RNA was isolated from rapidly frozen LV tissues using the SV Total RNA Isolation System (Promega, Madison, WI, USA). RNA was reverse transcribed by standard methods using a First-Strand cDNA Synthesis Kit (GE Healthcare UK Ltd). The expressions of type I and type III collagen, transforming growth factor (TGF)- $\beta 1$ and $-\beta 2$, angiotensin II type- $1\left(\mathrm{AT}_{1}\right)$ receptor $\left(\mathrm{AT}_{1} \mathrm{R}\right)$ and GAPDH were evaluated by real-time reverse transcription-PCR as described earlier. ${ }^{24}$ To correct the efficiency of cDNA synthesis, the amount of each measured mRNA was normalized to that of GAPDH mRNA as a housekeeping gene.

\section{Statistical analysis}

Quantitative results are expressed as mean \pm s.d. The serial data were analyzed by two-way analysis of variance, and the differences at specific stages among the groups were determined by Fisher's post hoc test. Differences in a single parameter among the five groups were evaluated using one-way analysis of variance followed by Fisher's post hoc test for multiple comparisons. The correlations between variables were assessed by linear regression analysis. Values of $P<0.05$ were considered to be statistically significant. 


\section{RESULTS}

\section{Blood pressure}

At 7 weeks of age, systolic blood pressure was comparable among the five groups (Table 1). At 17 weeks of age, after 10 weeks of a high-salt diet, the HT and HT+DM groups showed a comparable level of elevated blood pressure as compared with that in the control group, whereas the tail-cuff systolic blood pressure was equally elevated in the olmesartan group at the present dose (Table 1).

\section{Blood glucose levels}

Blood glucose levels at baseline were comparable among the five groups (Table 1). After STZ injection, all three STZ-treated groups (the DM, HT+DM and olmesartan groups) showed equally elevated glucose levels than in the control group (Table 1).

\section{Echocardiographic parameters}

In the HT and HT+DM groups, LV wall thickness was significantly increased at 17 weeks of age as compared with that in the control group (Figure 1). LVDd and fractional shortening were comparable in all groups, indicating that the LV systolic function was preserved in all groups (Figure 1). By contrast, the E/A ratio was significantly decreased in the HT group at 17 weeks of age, compared with that in the control group, and this decrease was significantly exaggerated in the $\mathrm{HT}+\mathrm{DM}$ group, indicating that, when superimposed on HT, DM more severely impaired the LV diastolic function without affecting the systolic function (Figure 1, Table 2). In the olmesartan group, as compared with the HT+DM group, LV wall thickness was significantly ameliorated without altering the tail-cuff systolic blood pressure, and
LV fractional shortening was slightly but significantly improved (Figure 1). The E/A ratio was significantly higher in the olmesartan group than in the HT+DM group (Figure 1).

\section{Hemodynamics}

At 17 weeks of age, peak positive $\mathrm{dP} / \mathrm{dt}$ was comparable among the five groups, indicating that there was no significant difference in $\mathrm{LV}$ systolic function among the five groups (Table 1). Peak negative $\mathrm{dP} / \mathrm{dt}$ and $\tau$, both of which are indices of LV relaxation, ${ }^{19,23,29}$ were significantly impaired in the $\mathrm{HT}, \mathrm{DM}$ and $\mathrm{HT}+\mathrm{DM}$ groups as compared with those in the control group, and this impairment was significantly exaggerated in the HT+DM group than in the HT group in terms of $\tau$ (Table 1). Although the LV end-diastolic pressure was within the normal range in all groups, it was significantly higher in the HT and HT+DM groups than in the control group (Table 1). The treatment with olmesartan significantly improved both peak negative $\mathrm{dP} / \mathrm{dt}$ and $\tau$, but not the LV end-diastolic pressure, as compared with those in the HT+DM group (Table 1).

\section{Organ weights and histology}

Body weight was significantly lower in the HT, DM, HT+DM and olmesartan groups than in the control group (Table 1). By contrast, the tibial length was comparable in all groups (Table 1). The ratio of LV to body weight or of LV to tibial length, an index of LV hypertrophy, and cardiomyocyte cross-sectional diameter were all significantly increased in the HT and $\mathrm{HT}+\mathrm{DM}$ groups as compared with those in the control group, although these two parameters were comparable between the HT and HT+DM groups (Table 1, Figure 2).

Table 1 Blood glucose, hemodynamic data and organ weights

\begin{tabular}{|c|c|c|c|c|c|}
\hline Groups & Control & $H T$ & $D M$ & $H T+D M$ & Olmesartan $(H T+D M+O l m e)$ \\
\hline \multicolumn{6}{|l|}{ Systolic blood pressure ( $\mathrm{mm} \mathrm{Hg}$ ) } \\
\hline$n$ & 14 & 14 & 12 & 12 & 11 \\
\hline 7 weeks of age & $126 \pm 16$ & $125 \pm 17$ & $135 \pm 13$ & $131 \pm 16$ & $129 \pm 8$ \\
\hline 17 weeks of age & $136 \pm 11$ & $209 \pm 18^{* *}$ & $126 \pm 17$ & $215 \pm 33^{* *}$ & $216 \pm 24^{* *}$ \\
\hline \multicolumn{6}{|l|}{ Blood glucose (mg per $100 \mathrm{ml}$ ) } \\
\hline$n$ & 14 & 14 & 12 & 12 & 12 \\
\hline Before STZ injection & $81 \pm 11$ & $85 \pm 10$ & $88 \pm 15$ & $80 \pm 7$ & $94 \pm 7^{* *}$ \\
\hline After STZ injection & $78 \pm 10$ & $88 \pm 11$ & $368 \pm 91^{* *, \ddagger}$ & $367 \pm 68^{* *, \frac{\ddagger}{\ddagger}}$ & $325 \pm 57 * *, \frac{\ddagger}{i}$ \\
\hline \multicolumn{6}{|l|}{ Hemodynamic data } \\
\hline$n$ & 14 & 14 & 11 & 10 & 9 \\
\hline Heart rate (beats $\mathrm{min}^{-1}$ ) & $241 \pm 35$ & $232 \pm 29$ & $214 \pm 30$ & $213 \pm 23$ & $216 \pm 24$ \\
\hline LVEDP $(\mathrm{mm} \mathrm{Hg})$ & $4.4 \pm 1.4$ & $6.4 \pm 2.1^{* *}$ & $5.7 \pm 2.4$ & $5.9 \pm 0.9 *$ & $9.1 \pm 1.5^{* *,+, \# \#}$ \\
\hline Peak positive $\mathrm{dP} / \mathrm{dt}\left(\mathrm{mm} \mathrm{Hg} \mathrm{s}^{-1}\right)$ & $7681 \pm 1483$ & $8191 \pm 1815$ & $7424 \pm 1231$ & $7886 \pm 1263$ & $8488 \pm 1517$ \\
\hline Peak negative $\mathrm{dP} / \mathrm{dt}\left(\mathrm{mm} \mathrm{Hg} \mathrm{s}^{-1}\right)$ & $8848 \pm 2366$ & $7063 \pm 1805^{* *}$ & $7386 \pm 1226^{*}$ & $6376 \pm 1383^{* *}$ & $7990 \pm 1008^{\#}$ \\
\hline$\tau(\mathrm{ms})$ & $13 \pm 1$ & $17 \pm 2 * *$ & $15 \pm 1 *$ & $18 \pm 1^{* *, \dagger}$ & 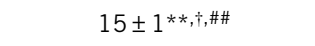 \\
\hline \multicolumn{6}{|l|}{ Organ weights } \\
\hline$n$ & 13 & 14 & 12 & 11 & 11 \\
\hline Final BW (g) & $384 \pm 24$ & $360 \pm 35^{*}$ & $352 \pm 25 * *$ & $342 \pm 26^{* *}$ & $338 \pm 28 * *$ \\
\hline Final TL (mm) & $57 \pm 2$ & $58 \pm 2$ & $57 \pm 1$ & $56 \pm 1$ & $57 \pm 1$ \\
\hline LV/BW $\left(\mathrm{mgg}^{-1}\right)$ & $2.35 \pm 0.16$ & $3.53 \pm 0.48^{* *}$ & $2.32 \pm 0.14$ & $3.54 \pm 0.27^{* *}$ & $3.50 \pm 0.39 * *$ \\
\hline Lung/BW (mgg $\left.{ }^{-1}\right)$ & $4.6 \pm 0.6$ & $5.2 \pm 0.5^{* *}$ & $4.9 \pm 0.3$ & $5.3 \pm 0.4 * *$ & $5.0 \pm 0.5^{*}$ \\
\hline $\mathrm{LV} / \mathrm{TL}\left(\mathrm{mg} \mathrm{mm} \mathrm{m}^{-1}\right)$ & $16 \pm 1$ & $22 \pm 2^{* *}$ & $15 \pm 1$ & $21 \pm 2 * *$ & $20 \pm 2^{* *, \dagger}$ \\
\hline Lung/TL $\left(\mathrm{mg} \mathrm{mm}^{-1}\right)$ & $30 \pm 3$ & $32 \pm 2$ & $31 \pm 2$ & $32 \pm 2$ & $29 \pm 2, \dagger, \#$ \\
\hline
\end{tabular}

Abbreviations: HT, hypertension; DM, diabetes mellitus; Olme, olmesartan; STZ, streptozotocin; LVEDP, left ventricular (LV) end-diastolic pressure; peak positive and negative dP/dt, peak positive and negative value of the first derivative of LV pressure; $\tau$, time constant of LV relaxation; BW, body weight; TL, tibial length.

and negative value of the first derivative

Results are expressed as mean $\pm \mathrm{s}$.
$n$ indicates the number of animals.

${ }^{*} P<0.05,{ }^{* *} P<0.01$ vs. Control; ${ }^{\dagger} P<0.05,{ }^{\ddagger} P<0.01$ vs. $\mathrm{HT} ;{ }^{\#} P<0.05,{ }^{\#} P<0.01$ vs. HT+DM 

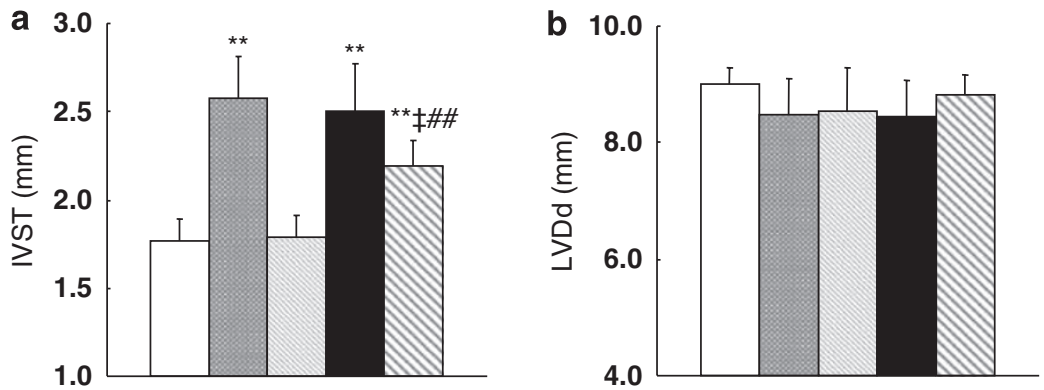

$\square$ Control $(n=14), \quad \square H T(n=14), \quad \square D M(n=11-12), \quad \square H T+D M(n=12), \quad \square H T+D M+$ Olme $(n=11)$
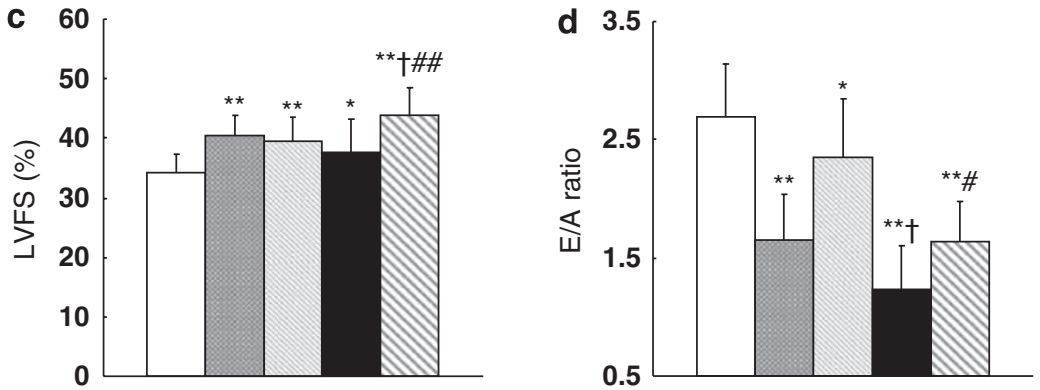

Figure 1 Effects of diabetes mellitus on left ventricular hypertrophy, systolic function and diastolic dysfunction. Quantitative measurement of interventricular septum thickness (IVST) (a), left ventricular (LV) diameter at end diastole (LVDd) (b), LV fractional shortening (LVFS) (c) and E/A ratio (d) on transthoracic Mmode and Doppler echocardiographic studies in the control, hypertension (HT), diabetes mellitus (DM), untreated HT+DM and HT+DM treated with olmesartan ( $1 \mathrm{mg} \mathrm{kg}^{-1} \mathrm{day}^{-1}$, p.o.) from 11 weeks of age (HT+DM+Olme) groups at 17 weeks of age. DM had no additive deleterious effects on LV hypertrophy (IVST) and systolic function (LVDd, LVFS) in the present model. By contrast, olmesartan treatment significantly ameliorated LV hypertrophy and systolic function. Diastolic dysfunction (the decrease in E/A ratio) was synergistically induced by DM, together with hypertension, and was significantly improved by olmesartan. Results are expressed as mean \pm s.d. ${ }^{*} P<0.05,{ }^{*} P<0.01$ vs. Control; ${ }^{\dagger} P<0.05,{ }^{\ddagger} P<0.01$ vs. HT; ${ }^{\#} P<0.05, \# \# P<0.01$ vs. HT + DM.

Table 2 Other echocardiographic parameters

\begin{tabular}{|c|c|c|c|c|c|}
\hline Groups & Control & $H T$ & $D M$ & $H T+D M$ & $\begin{array}{c}\text { Olmesartan } \\
\text { (HT+DM+ } \\
\text { Olme) }\end{array}$ \\
\hline \multicolumn{6}{|c|}{ Echocardiographic parameters } \\
\hline$n$ & 14 & 14 & 11 & 12 & 11 \\
\hline $\begin{array}{l}\text { Heart rate } \\
\text { (beats } \text { min }^{-1} \text { ) }\end{array}$ & $243 \pm 13$ & $247 \pm 33$ & $218 \pm 16^{* *, \ddagger}$ & $243 \pm 23$ & 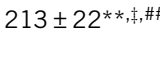 \\
\hline $\begin{array}{l}\text { Peak E velocity } \\
\left(\mathrm{cm} \mathrm{s}^{-1}\right)\end{array}$ & $92 \pm 11$ & $90 \pm 16$ & $100 \pm 10$ & $73 \pm 18^{* *, \ddagger}$ & $90 \pm 10^{\# \#}$ \\
\hline $\begin{array}{l}\text { Peak A velocity } \\
\left(\mathrm{cm} \mathrm{s}^{-1}\right)\end{array}$ & $35 \pm 5$ & $58 \pm 16^{* *}$ & * $45 \pm 11^{\dagger}$ & $62 \pm 16^{* *}$ & $59 \pm 18 * *$ \\
\hline
\end{tabular}

The ratio of lung to body weight, an index of left-sided heart failure, was slightly but significantly increased in the HT and HT+DM groups to a similar extent as compared with that in the control group (Table 1). Myocardial interstitial fibrosis in the LV was significantly increased in the HT group and, to a greater extent, in the HT+DM group (Figure 3). Olmesartan treatment significantly ameliorated cardiomyocyte cardiomyocyte cross-sectional diameter and myocardial interstitial fibrosis as compared with those in the HT+DM group (Figures 2 and 3), although it did not improve the ratio of LV to body weight (Table 1). Olmesartan also tended to decrease the ratio of lung to body weight $(P=0.08)$ (Table 1$)$.

\section{Collagen gene expression}

The HT+DM group showed an increased mRNA expression of type III collagen, but not of type I collagen, and these upregulations were significantly ameliorated by olmesartan treatment (Figures $4 \mathrm{a}$ and b).

\section{Proinflammatory cytokines}

The mRNA expression of TGF- $\beta 1$ and of TGF- $\beta 2$ was enhanced in the $\mathrm{HT}$ and $\mathrm{HT}+\mathrm{DM}$ groups as compared with that in the control group, wherein the olmesartan treatment significantly suppressed the expression of TGF- $\beta 2$ and of TGF- $\beta 1$ ( $P=0.05$, Figures $4 \mathrm{c}$ and $d)$.

\section{Cardiac oxidative stress}

Cardiac 4-hydroxy-2-nonenal-modified protein expression, a marker of reactive oxygen species production, ${ }^{30}$ was significantly increased in the DM and HT+DM groups as compared with that in the control and HT groups, indicating that hyperglycemia enhanced cardiac oxidative stress (Figure 5a). Olmesartan treatment significantly ameliorated the 4-hydroxy-2-nonenal level (Figure 5a).

\section{Cardiac $\mathrm{ACE}$ and $\mathrm{AT}_{1} \mathrm{R}$ expression}

Cardiac ACE expression and $\mathrm{AT}_{1} \mathrm{R}$ mRNA levels were significantly increased in the HT+DM group than in the control, HT and DM groups, indicating that the combination of HT and DM significantly activated cardiac RAS (Figures $5 b$ and $6 a$ ). These enhanced ACE and $\mathrm{AT}_{1} \mathrm{R}$ expressions were significantly suppressed by olmesartan treatment (Figures $5 \mathrm{~b}$ and $6 \mathrm{a}$ ). Importantly, there was a positive correlation between the extent of cardiac ACE expression and that of diastolic dysfunction (negative $\mathrm{dP} / \mathrm{dt}$ and $\tau$ ), as well as between the $\mathrm{AT}_{1} \mathrm{R}$ mRNA levels and $\tau$ (Figures $5 c, d$ and $6 \mathrm{~b}$ ). 
a

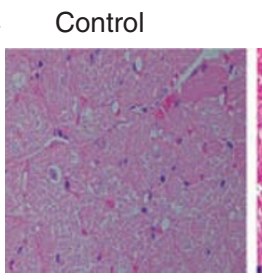

HT

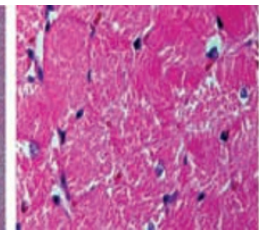

b
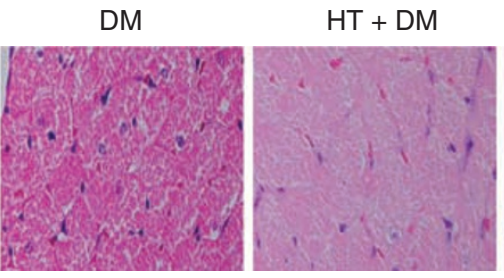

$\mathrm{HT}+\mathrm{DM}+\mathrm{Olme}$

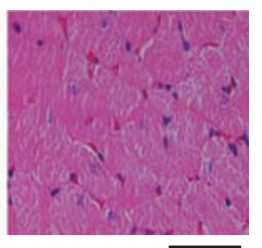

$\overline{50 \mu \mathrm{m}}$

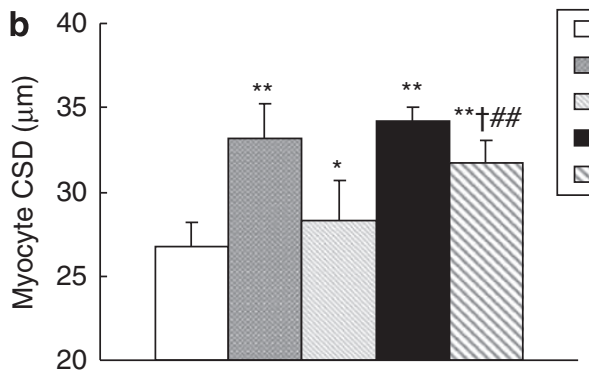

$\square$ Control $(n=14)$

$\square \mathrm{HT}(\mathrm{n}=14)$

$\square \mathrm{DM}(\mathrm{n}=12)$

- HT + DM $(n=11)$

$\checkmark H T+D M+$ Olme $(n=11)$

Figure 2 Effect of diabetes mellitus on cardiomyocyte hypertrophy. (a) Representative photomicrographs of cardiomyocytes from rats in the control, HT, DM, untreated HT+DM and HT+DM treated with olmesartan ( $1 \mathrm{mg} \mathrm{kg}^{-1}$ day ${ }^{-1}$, p.o.) from 11 weeks of age (HT+DM+Olme) groups at 17 weeks of age (hematoxylin and eosin staining, $\times 400$ ). (b) Quantitative analysis of cardiomyocyte hypertrophy. CSD, cross-sectional diameter of cardiomyocyte. CSD was significantly increased in the HT and HT+DM groups; however, there was no significant difference in this parameter between the two groups. Cardiomyocyte hypertrophy was significantly suppressed by olmesartan. Results are expressed as mean \pm s.d. ${ }^{*} P<0.05,{ }^{* *} P<0.01$ vs. Control; ${ }^{\dagger} P<0.05$ vs. HT; $\#$ \# $<0.01$ vs. HT + DM.

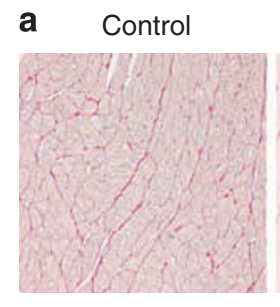

HT

DM

$\mathrm{HT}+\mathrm{DM}$

$\mathrm{HT}+\mathrm{DM}+$ Olme
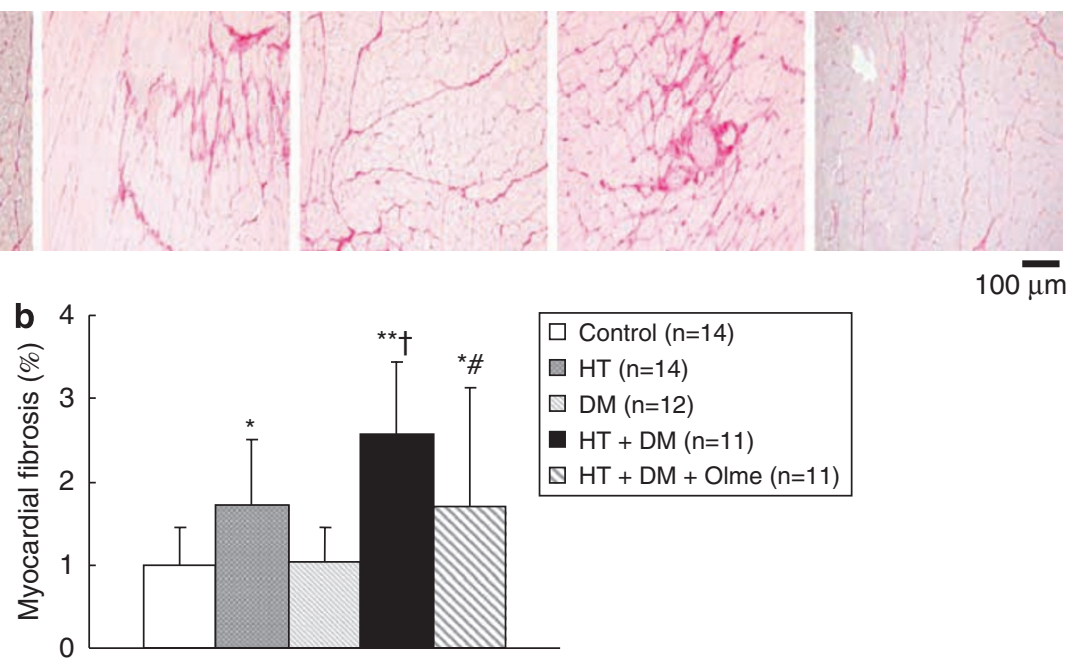

$100 \overline{\mu m}$

Figure 3 Effect of diabetes mellitus on cardiac interstitial fibrosis. (a) Representative photomicrographs of the myocardium in the control, HT, DM, untreated $\mathrm{HT}+\mathrm{DM}$ and $\mathrm{HT}+\mathrm{DM}$ treated with olmesartan $\left(1 \mathrm{mg} \mathrm{kg}^{-1}\right.$ day $^{-1}$, p.o.) from 11 weeks of age (HT+DM+Olme) groups at 17 weeks of age (sirius red staining, $\times 100$ ). (b) Quantitative analysis of myocardial fibrosis. DM synergistically increased LV interstitial fibrosis, together with hypertension. Myocardial fibrosis was significantly suppressed by olmesartan. Results are expressed as mean \pm s.d. ${ }^{*} P<0.05,{ }^{* *} P<0.01$ vs. Control; ${ }^{\dagger} P<0.05$ vs. HT; ${ }^{\#} P<0.05$ vs. HT+DM.

\section{DISCUSSION}

The novel findings of this study are that (1) the association of DM with HT significantly activates cardiac RAS in a rat model of pure diastolic dysfunction, resulting in an increase in LV interstitial fibrosis, and further accelerates LV diastolic dysfunction, and (2) the inhibition of RAS with olmesartan ameliorates the HT+DM-induced diastolic dysfunction, interstitial fibrosis and the RAS activation of LV, independent of the changes in tail-cuff systolic blood pressure measured at 2-week intervals, as vasodilators including olmesartan might not decrease blood pressure in this volume-overloaded HT model at lower doses. ${ }^{19}$ To the best of our knowledge, this is the first study that shows that an association of DM accelerates LV diastolic dysfunc- tion in the hypertensive heart through RAS activation and the resultant oxidative stress (Figure 7).

\section{Development of an animal model of pure diastolic dysfunction because of $\mathrm{HT}+\mathrm{DM}$}

Diabetes mellitus frequently coexists with HT as the concomitant underlying diseases in patients with DHF. ${ }^{6,7}$ It was suggested earlier that patients with both DM and HT have more severely impaired LV relaxation than those with either condition alone. ${ }^{11}$ However, little is known about the detailed mechanisms of the diastolic dysfunction associated with DM and HT. In this study, we developed the HT+DM model, in which a Dahl salt-sensitive rat is fed a high-salt $(5 \% \mathrm{NaCl})$ 

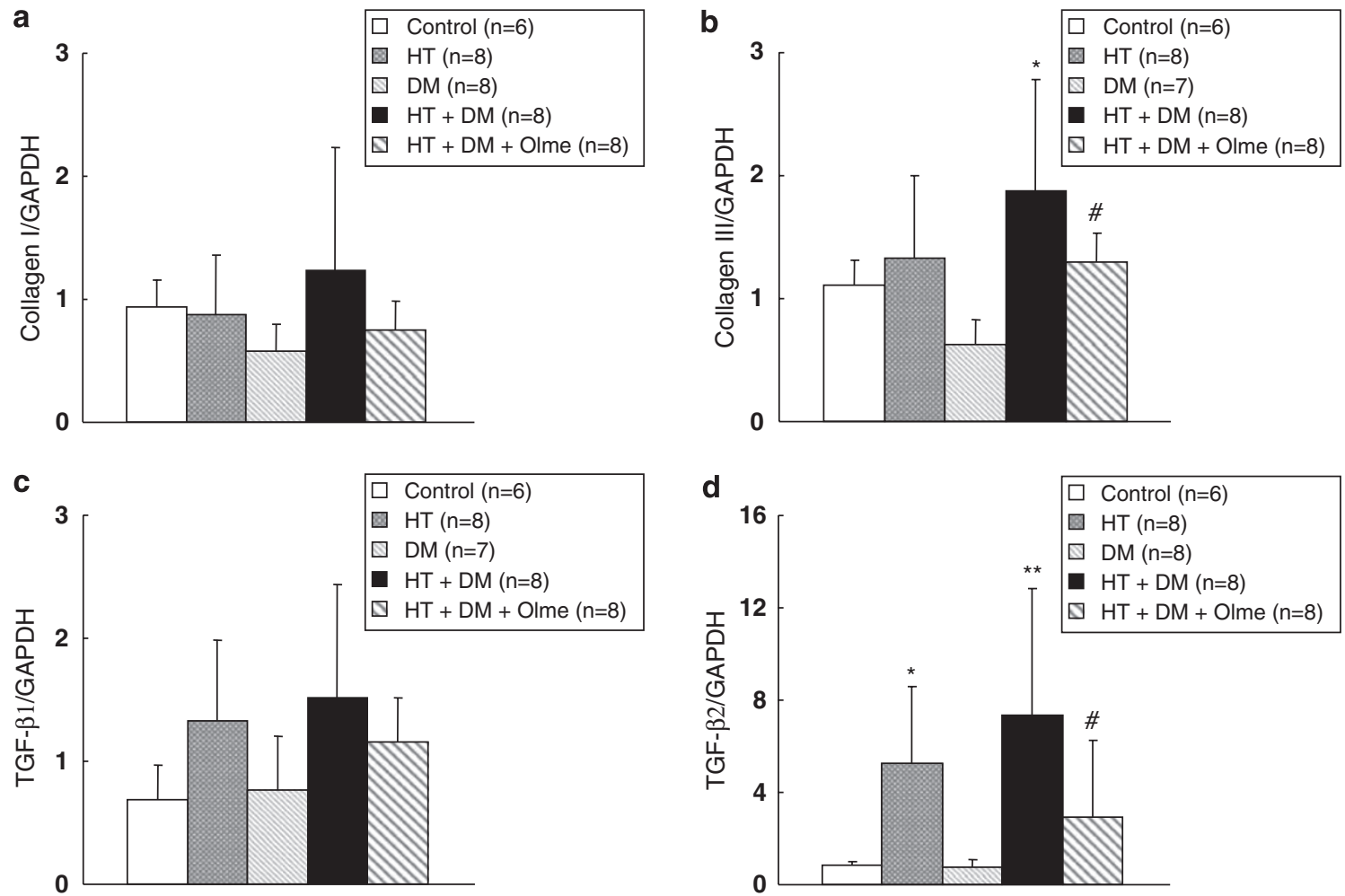

Figure 4 Effects of diabetes mellitus on collagen synthesis and inflammatory change. The mRNA level of type I collagen (a), type III collagen (b), transforming growth factor (TGF)- $\beta 1$ (c) and TGF $-\beta 2$ (d) in the control, HT, DM, untreated HT+DM and HT+DM treated with olmesartan (1 mg kg ${ }^{-1}$ day ${ }^{-1}$, p.o.) from 11 weeks of age (HT+DM+Olme) groups at 17 weeks of age. Collagen synthesis (type III collagen) and inflammatory change (TGF - $\beta 2$ ) were significantly enhanced in the HT+DM group, which were also significantly suppressed by olmesartan. Results are expressed as mean \pm s.d. ${ }^{*} P<0.05$, ${ }^{* *} P<0.01$ vs. Control; ${ }^{\#} P<0.05$ vs. HT+DM.
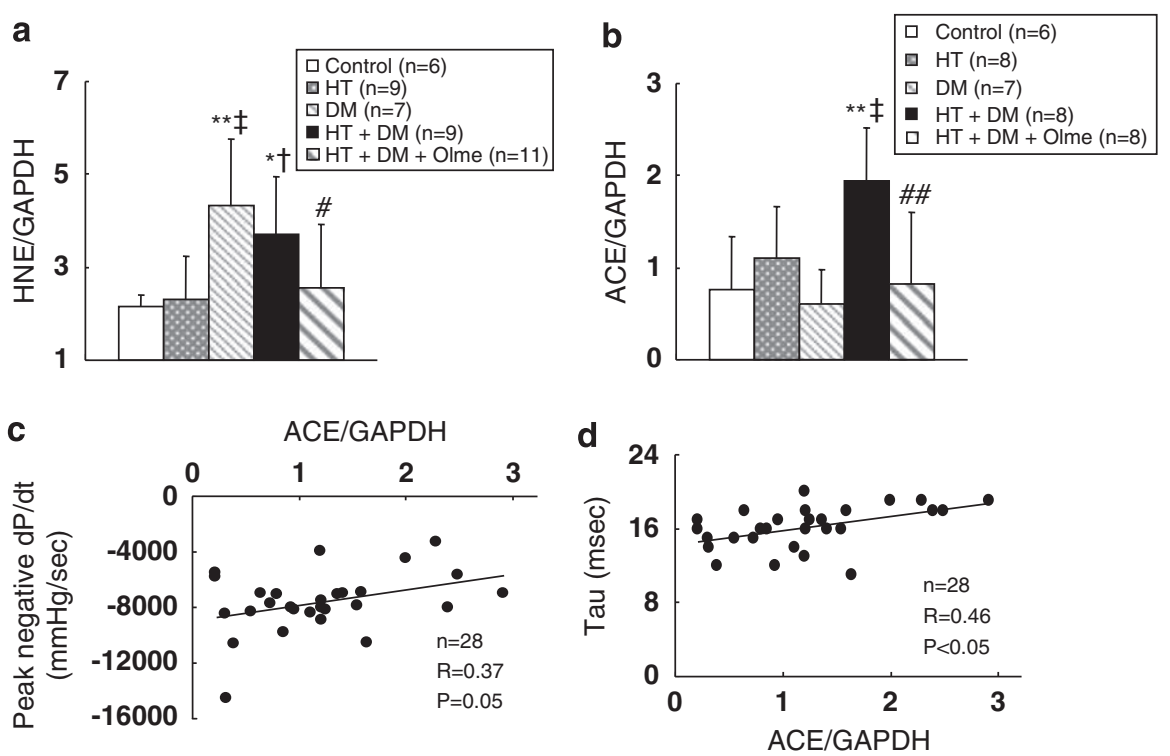

Figure 5 Effect of diabetes mellitus on cardiac oxidative stress and angiotensin-converting enzyme (ACE). (a) Quantitative analysis of western blot data for 4 hydroxy-2-nonenal (HNE)-modified protein and glyceraldehydes-3-phosphate-dehydrogenase (GAPDH) in the control, HT, DM, untreated HT+DM and HT+DM treated with olmesartan ( $1 \mathrm{mg} \mathrm{kg}^{-1}$ day $^{-1}$, p.o.) from 11 weeks of age (HT+DM+Olme) groups at 17 weeks of age. Hyperglycemia, but not hypertension, significantly enhanced myocardial oxidative stress, as evaluated by the ratio of HNE/GAPDH, which was significantly suppressed by olmesartan. (b) Quantitative analysis of western blot data for angiotensin-converting enzyme (ACE) and GAPDH in the control, HT, DM and untreated HT+DM groups at 17 weeks of age. DM synergistically activated the cardiac rennin-angiotensin system (RAS), as evaluated by the ratio of ACE/GAPDH, when superimposed on hypertension. Cardiac RAS activation was again significantly suppressed by olmesartan. (c and $\mathbf{d})$ There was a significant positive correlation between the extent of cardiac ACE expression and that of diastolic dysfunction including both negative $\mathrm{dP} / \mathrm{dt}$ (c) and $\tau$ (d) from rats in the five groups. ${ }^{*} P<0.05$, ${ }^{* *} P<0.01$ vs. Control; ${ }^{\dagger} P<0.05, \ddagger P<0.01$ vs. $\mathrm{HT} ;{ }^{\#} P<0.05$, $\# \#<<0.01$ vs. HT+DM. 

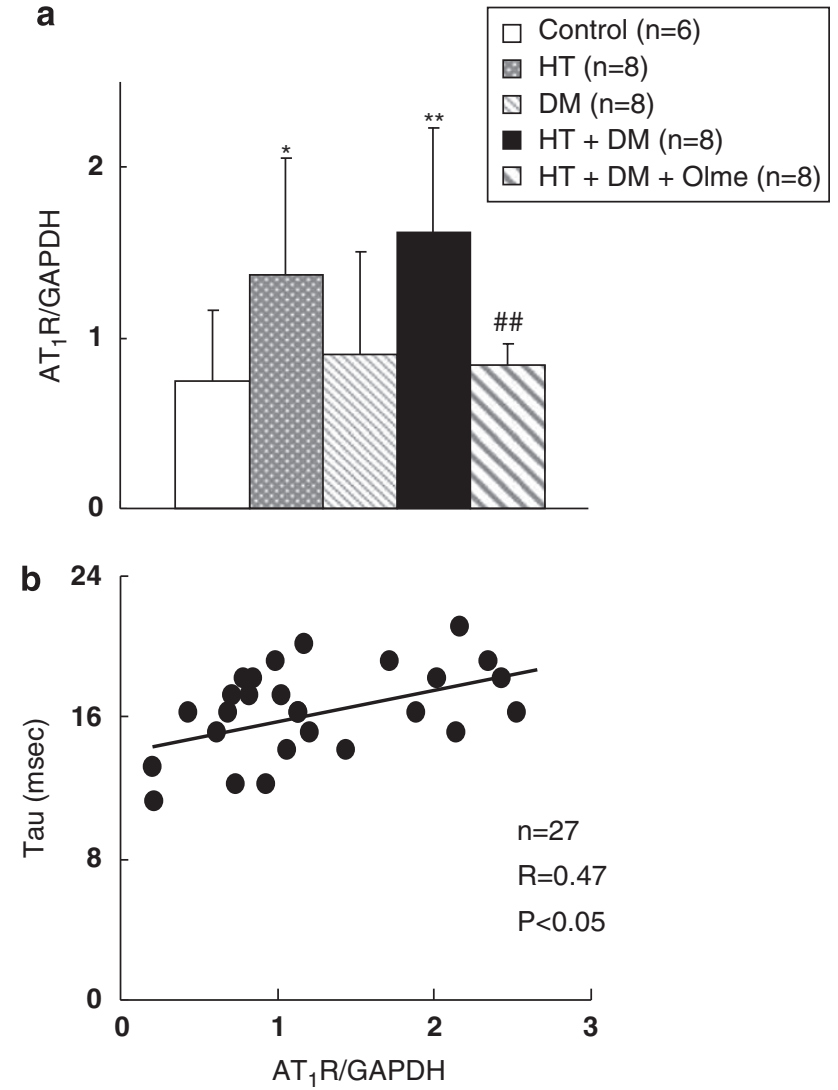

Figure 6 The effect of diabetes mellitus on mRNA levels of angiotensin II type 1 receptor. (a) The mRNA levels of angiotensin II type 1 receptor $\left(A T_{1} R\right)$ in the five groups at 17 weeks of age. DM synergistically activated the cardiac renin-angiotensin system (RAS), as evaluated by the ratio of $A T_{1} R / G A P D H$, when superimposed on hypertension. The cardiac $A T_{1} R$ expression was significantly suppressed by olmesartan. (b) There was a significant positive correlation between the mRNA levels of cardiac $A T_{1} R$ and $\tau$ from rats in the control, HT, DM and untreated HT+DM groups. Results are expressed as mean \pm s.d. ${ }^{*} P<0.05,{ }^{* *} P<0.01$ vs. Control; $\#$ \# $<0.01$ vs. HT+DM.

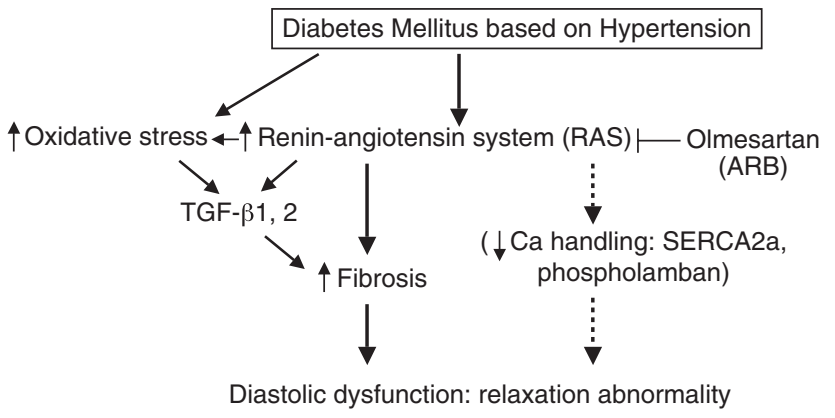

Figure 7 Summary of the present findings. The abnormalities of the left ventricle (LV) in Dahl salt-sensitive rat model with diabetes mellitus and hypertension are characterized by the activated renin-angiotensin system (RAS) and oxidative stress, which cause LV myocardial fibrosis and subsequent LV diastolic dysfunction.

diet for 10 weeks starting at 7 weeks of age, in combination with STZ injection at 11 weeks of age. All hypertensive and diabetic animals survived for 10 weeks without showing any heart failure symptoms or lung edema. We confirmed that at 17 weeks of age, the HT+DM group exhibited more severely impaired LV relaxation than did the HT group with preserved systolic function.

\section{Impact of DM on diastolic dysfunction, myocardial fibrosis and RAS activation}

In this study, although the extent of LV hypertrophy was comparable between the HT and HT+DM groups, as evidenced by both the ratio of LV weight to body weight and cardiomyocyte cross-sectional diameter, LV interstitial fibrosis was significantly enhanced in the latter group. As LV fibrosis plays a major role in the pathogenesis of LV diastolic dysfunction, ${ }^{31-33}$ the mechanisms of DM-induced acceleration of LV diastolic dysfunction can be explained, at least in part, by the increased LV interstitial fibrosis, but not by the additional LV hypertrophy. In this study, we were able to show that cardiac ACE and $\mathrm{AT}_{1} \mathrm{R}$ expressions were significantly increased in the $\mathrm{HT}+\mathrm{DM}$ group, indicating that DM complicated with HT markedly activates cardiac RAS in our model. This is consistent with a recent study showing that RAS is activated in DM. ${ }^{34}$ Earlier studies have shown that RAS plays an important role in ventricular fibrosis mainly through $\mathrm{AT}_{1} \mathrm{R}^{35-38}$ This study further shows that olmesartan treatment inhibits myocardial fibrosis and LV diastolic dysfunction induced by HT and DM.

This study also showed that the expression of TGF- $\beta$ was significantly enhanced in the HT and HT+DM groups. TGF- $\beta$ is known to be a major stimulator of myocardial fibrosis activated by angiotensin II. ${ }^{36}$ Thus, the fibrotic cytokine is likely to be involved in the pathogenesis of myocardial fibrosis in our present model. Moreover, reactive oxygen species production was significantly increased in the DM and HT+DM groups in this study. This is consistent with an earlier study that showed that hyperglycemia itself enhances oxidative stress. ${ }^{39}$ Oxidative stress, which is also activated by an $\mathrm{AT}_{1} \mathrm{R}$-mediated pathway ${ }^{40,41}$ and promotes myocardial fibrosis, ${ }^{15,42}$ is likely to be involved in the development of myocardial fibrosis in DM, as shown in this study. Furthermore, we were able to show that there was a positive correlation between the extent of cardiac ACE expression and that of diastolic dysfunction. Taken together, DM-induced RAS activation is substantially involved in the pathogenesis of LV diastolic dysfunction by promoting inflammatory changes, oxidative stress and the resultant interstitial fibrosis (Figure 7).

The left ventricular relaxation abnormality, an early manifestation of diabetic cardiomyopathy, is one of the most important determinants of diastolic dysfunction, ${ }^{43}$ and may contribute to the development of DHF. ${ }^{6} \mathrm{LV}$ relaxation is regulated by cytosolic $\mathrm{Ca}^{2+}$ handling, including SERCA2a and phospholamban, both of which are involved in $\mathrm{Ca}^{2+}$ re-uptake into the sarcoplasmic reticulum. Sakata et al. ${ }^{44}$ showed in a rat DHF model that phosphorylation levels of phospholamban may play a more important role in LV relaxation abnormality than does the protein level of SERCA2a or phospholamban. ${ }^{15,44}$ In that model, $\mathrm{AT}_{1} \mathrm{R}$-blockade ameliorated the downregulation of phosphorylated phospholamban and cAMP concentration, resulting in an improvement of the LV relaxation capacity. ${ }^{15,44}$ These results suggest a close relationship between RAS and LV relaxation. In addition, DM is directly associated with the abnormality of intracellular $\mathrm{Ca}^{2+}$ mobilization through enhanced free fatty acid turnover (for example, lipotoxicity). ${ }^{6,45}$ Thus, it is conceivable that this $\mathrm{Ca}^{2+}$ handling abnormality resulting from DM-induced RAS activation is also involved in the pathogenesis of the LV diastolic dysfunction in this study, in addition to an enhanced interstitial fibrosis (Figure 7).

\section{Beneficial effects of olmesartan on diastolic dysfunction}

Earlier studies have shown the beneficial effects of RAS inhibition in the development of diabetic complications including microangiopathy 
and macroangiopathy. ${ }^{46,47}$ In this study, olmesartan treatment significantly ameliorated LV diastolic dysfunction and interstitial fibrosis. We were also able to show that olmesartan ameliorated cardiomyocyte hypertrophy, increased collagen synthesis, and upregulated inflammatory cytokines. Moreover, olmesartan treatment significantly suppressed hyperglycemia-induced cardiac oxidative stress, suggesting the antioxidative effect of the $\mathrm{AT}_{1} \mathrm{R}$ antagonist. In addition, $\mathrm{AT}_{1} \mathrm{R}$ antagonists can inhibit the angiotensin II-induced upregulation of ACE mRNA, ${ }^{31,48}$ which is consistent with our present findings. Taken together, the beneficial effects of olmesartan on LV diastolic dysfunction are likely mediated, at least in part, by its direct inhibitory effects on cardiac RAS activation and oxidative stress, in addition to its antihypertensive effect.

\section{Limitations of the study}

Several limitations should be mentioned for this study. First, in this study, we used STZ-induced diabetic rats as an animal model of DM, which is considered as a model of type-1 DM. However, type-2 DM is the majority form of DM that causes cardiomyopathy in the clinical setting of hypertensive heart disease. Thus, the present rat model may not fully represent the clinical features of patients with hypertensive heart disease complicated with DM. Second, in this study, the DM alone group exhibited only a modest abnormality of cardiac function, which is probably because of the short duration and modest severity of hyperglycemia. Longer exposure to diabetes with higher glucose levels might have caused more severe oxidative stress and LV fibrosis, which may worsen hemodynamic and morphological parameters. Therefore, in future studies, we also need to clarify the further mechanisms whereby HT and DM interact to cause diastolic dysfunction. Third, in this study, the increased ACE expression in the HT alone group did not reach statistical significance, although several earlier studies showed that the rat model of DHF with HT was associated with a significant activation of cardiac RAS. ${ }^{31,35}$ This discrepancy can be explained by the differences in the amount of sodium intake. Indeed, the elevation of systolic blood pressure in those and in our previous studies was higher than that in this study, ${ }^{19,35}$ because Dahl salt-sensitive rats were fed a diet containing $8 \% \mathrm{NaCl}$ in those earlier studies. Moreover, in this study, we only assessed cardiac $\mathrm{AT}_{1} \mathrm{R}$ mRNA levels and protein levels of cardiac ACE, which may not necessarily reflect RAS activity. Fourth, in this study, we used a tail-cuff method to measure blood pressure, which is incapable of detecting the changes in diastolic blood pressure, in $24 \mathrm{~h}$ blood pressure or in blood pressure at night. Therefore, we cannot completely exclude the possibility that the olmesartan treatment might have decreased these blood pressures, accounting for its cardioprotective effects observed in this study. Furthermore, it is conceivable that these protective effects associated with olmesartan treatment could be caused by its blood pressurelowering effects which could not be detected by the tail-cuff method. Fifth, as all echocardiographic and hemodynamic measurements were determined under general anesthesia, we cannot completely exclude the influence of anesthesia in these results. However, anesthesia was carried out in the same manner in all the five groups. Sixth, we did not examine the change in insulin sensitivity in this study, which may influence diabetic cardiomyopathy and be affected by olmesartan treatment. ${ }^{49}$ However, blood glucose levels at 17 weeks of age were comparable in the untreated $\mathrm{HT}+\mathrm{DM}$ and olmesartan groups ( $407 \pm 234$ vs. $489 \pm 172 \mathrm{mg}$ per $100 \mathrm{ml}, P=0.25$ ). Finally, heart rates in the echocardiographic study were significantly lower in two out of three diabetic groups (DM and olmesartan groups) as compared with those in the other groups (Table 2). As the values of Doppler echocardiographic index (E/A ratio) can be influenced by lower heart rates, ${ }^{50}$ we have to take into account its effects on LV relaxation capacity. However, in this study, the E/A ratio was not correlated with the heart rates obtained in the echocardiographic study in all the five groups $(R=0.10, P=0.44)$. Moreover, we further evaluated the $\mathrm{LV}$ relaxation capacity not only with the echo-Doppler study but also with the hemodynamic study using Miller's catheter, in which there was no significant difference in heart rates among the five groups (Table 1). Therefore, we consider that our evaluation of LV relaxation was appropriate and correct, although we cannot completely exclude the influence of heart rate.

\section{Clinical implications}

This study indicates that an association of DM with HT accelerates the LV diastolic dysfunction mainly through RAS activation and oxidative stress, suggesting that RAS inhibition is useful for the treatment of hypertensive heart disease complicated with DM in humans.

\section{CONFLICT OF INTEREST}

The authors declare no conflict of interest.

\section{ACKNOWLEDGEMENTS}

We are grateful to Fumie Hase and Naomi Yamaki for their excellent technical assistance in this study. We also thank Daiichi Sankyo Co. Ltd, Tokyo, Japan, for providing olmesartan. This work was supported by the grant-in-aid for Scientific Research C (no. 19590803 to Dr Fukumoto) and in part by the grants-in-aid (nos. 15256003, 16209027, 16659192 to Dr Shimokawa) from the Japanese Ministry of Education, Culture, Sports, Science and Technology, Tokyo, Japan, the Program for Promotion of Fundamental Studies in Health Sciences of the Organization for Pharmaceutical Safety, and Research of Japan, Tokyo, Japan, Technology Agency, CREST, Tokyo, Japan, and the Japan Foundation of Cardiovascular Research, Tokyo, Japan.

1 Hunt SA, Abraham WT, Chin MH, Feldman AM, Francis GS, Ganiats TG, Jessup M, Konstam MA, Mancini DM, Michl K, Oates JA, Rahko PS, Silver MA, Stevenson LW, Yancy CW, Antman EM, Smith Jr SC, Adams CD, Anderson JL, Faxon DP, Fuster V, Halperin JL, Hiratzka LF, Jacobs AK, Nishimura R, Ornato JP, Page RL, Riegel B. ACC/ AHA 2005 Guideline update for the diagnosis and management of chronic heart failure in the adult: a report of the American College of Cardiology/American Heart Association Task Force on Practice Guidelines. Circulation 2005; 112: e154-e235.

2 Redfield MM, Jacobsen SJ, Burnett Jr JC, Mahoney DW, Bailey KR, Rodeheffer RJ. Burden of systolic and diastolic ventricular dysfunction in the community: appreciating the scope of the heart failure epidemic. JAMA 2003; 289: 194-202.

3 Kitzman DW, Little WC, Brubaker PH, Anderson RT, Hundley WG, Marburger CT, Brosnihan B, Morgan TM, Stewart KP. Pathophysiological characterization of isolated diastolic heart failure in comparison to systolic heart failure. JAMA 2002; 288: 2144-2150.

4 Cleland JG, Cohen-Solal A, Aguilar JC, Dietz R, Eastaugh J, Follath F, Freemantle N, Gavazzi A, van Gilst WH, Hobbs FD, Korewicki J, Madeira HC, Preda I, Swedberg K, Widimsky J. Management of heart failure in primary care (the IMPROVEMENT of Heart Failure Programme): an international survey. Lancet 2002; 360: 1631-1639.

5 Bhatia RS, Tu JV, Lee DS, Austin PC, Fang J, Haouzi A, Gong Y, Liu PP. Outcome of heart failure with preserved ejection fraction in a population-based study. $N \mathrm{Eng} / \mathrm{J}$ Med 2006; 355: 260-269.

6 Piccini JP, Klein L, Gheorghiade M, Bonow RO. New insights into diastolic heart failure: role of diabetes mellitus. Am J Med 2004; 116 (Suppl 5A): 64S-75S.

7 O'Connor CM, Gattis WA, Shaw L, Cuffe MS, Califf RM. Clinical characteristics and long-term outcomes of patients with heart failure and preserved systolic function. Am J Cardiol 2000; 86: 863-867.

8 Parker AB, Yusuf S, Naylor CD. The relevance of subgroup-specific treatment effects: the Studies Of Left Ventricular Dysfunction (SOLVD) revisited. Am Heart J 2002; 144: 941-947.

9 Mihm MJ, Seifert JL, Coyle CM, Bauer JA. Diabetes related cardiomyopathy time dependent echocardiographic evaluation in an experimental rat model. Life Sci 2001; 69: 527-542.

10 Hoit BD, Castro C, Bultron G, Knight S, Matlib MA. Noninvasive evaluation of cardiac dysfunction by echocardiography in streptozotocin-induced diabetic rats. J Card Fail 1999; 5: 324-333.

11 Liu JE, Palmieri V, Roman MJ, Bella JN, Fabsitz R, Howard BV, Welty TK, Lee ET, Devereux RB. The impact of diabetes on left ventricular filling pattern in normotensive 
and hypertensive adults: the Strong Heart Study. J Am Coll Cardiol 2001; 37: 1943-1949.

12 Shiomi T, Tsutsui H, Ikeuchi M, Matsusaka H, Hayashidani S, Suematsu N, Wen J, Kubota T, Takeshita A. Streptozotocin-induced hyperglycemia exacerbates left ventricular remodeling and failure after experimental myocardial infarction. J Am Coll Cardiol 2003; 42: 165-172.

13 Matsusaka H, Kinugawa S, Ide T, Matsushima S, Shiomi T, Kubota T, Sunagawa K, Tsutsui $\mathrm{H}$. Angiotensin II type 1 receptor blocker attenuates exacerbated left ventricular remodeling and failure in diabetes-associated myocardial infarction. J Cardiovasc Pharmacol 2006; 48: 95-102.

14 Given MB, Lowe RF, Gelvin CR, Sander GE, Giles TD. Preservation of left ventricular function and coronary flow by angiotensin I-converting enzyme inhibition in the hypertensive-diabetic Dahl rat. Am J Hypertens 1994; 7: 919-925.

15 Nishio M, Sakata Y, Mano T, Yoshida J, Ohtani T, Takeda Y, Miwa T, Masuyama T, Yamamoto K, Hori M. Therapeutic effects of angiotensin II type 1 receptor blocker at an advanced stage of hypertensive diastolic heart failure. J Hypertens 2007; 25: $455-461$.

16 Inagaki K, Koyanagi T, Berry NC, Sun L, Mochly-Rosen D. Pharmacological inhibition of epsilon-protein kinase $\mathrm{C}$ attenuates cardiac fibrosis and dysfunction in hypertensioninduced heart failure. Hypertension 2008; 51: 1565-1569.

17 Hoshi-Fukushima R, Nakamoto H, Imai H, Kanno Y, Ishida Y, Yamanouchi Y, Suzuki H. Estrogen and angiotensin II interactions determine cardio-renal damage in Dahl saltsensitive rats with heart failure. Am J Nephrol 2008; 28: 413-423.

18 Asaumi Y, Kagaya Y, Takeda M, Yamaguchi N, Tada H, Ito K, Ohta J, Shiroto T, Shirato $\mathrm{K}$, Minegishi N, Shimokawa H. Protective role of endogenous erythropoietin system in nonhematopoietic cells against pressure overload-induced left ventricular dysfunction in mice. Circulation 2007; 115: 2022-2032.

19 Fukui S, Fukumoto Y, Suzuki J, Saji K, Nawata J, Tawara S, Shinozaki T, Kagaya Y, Shimokawa H. Long-term inhibition of Rho-kinase ameliorates diastolic heart failure in hypertensive rats. J Cardiovasc Pharmacol 2008; 51: 317-326.

20 Doi R, Masuyama T, Yamamoto K, Doi Y, Mano T, Sakata Y, Ono K, Kuzuya T, Hirota S, Koyama T, Miwa T, Hori M. Development of different phenotypes of hypertensive heart failure: systolic versus diastolic failure in Dahl salt-sensitive rats. J Hypertens 2000; 18: $111-120$.

21 Sahn DJ, DeMaria A, Kisslo J, Weyman A. Recommendations regarding quantitation in M-mode echocardiography: results of a survey of echocardiographic measurements. Circulation 1978; 58: 1072-1083.

22 Masuyama T, Yamamoto K, Sakata Y, Doi R, Nishikawa N, Kondo H, Ono K, Kuzuya T, Sugawara M, Hori M. Evolving changes in Doppler mitral flow velocity pattern in rats with hypertensive hypertrophy. J Am Coll Cardiol 2000; 36: 2333-2338.

23 Yamamoto K, Masuyama T, Doi Y, Naito J, Mano T, Kondo H, Nagano R, Tanouchi J, Hori M, Kamada T. Noninvasive assessment of left ventricular relaxation using continuous-wave Doppler aortic regurgitant velocity curve. Its comparative value to the mitral regurgitation method. Circulation 1995; 91: 192-200.

24 Higashi M, Shimokawa H, Hattori T, Hiroki J, Mukai Y, Morikawa K, Ichiki T, Takahashi $\mathrm{S}$, Takeshita A. Long-term inhibition of Rho-kinase suppresses angiotensin II-induced cardiovascular hypertrophy in rats in vivo: effect on endothelial NAD(P)H oxidase system. Circ Res 2003; 93: 767-775.

25 Fukumoto Y, Libby P, Rabkin E, Hill CC, Enomoto M, Hirouchi Y, Shiomi M, Aikawa M. Statins alter smooth muscle cell accumulation and collagen content in established atheroma of watanabe heritable hyperlipidemic rabbits. Circulation 2001; 103: 993-999.

26 Fukumoto Y, Deguchi JO, Libby P, Rabkin-Aikawa E, Sakata Y, Chin MT, Hill CC, Lawler PR, Varo N, Schoen FJ, Krane SM, Aikawa M. Genetically determined resistance to collagenase action augments interstitial collagen accumulation in atherosclerotic plaques. Circulation 2004; 110: 1953-1959.

27 Kitamura M, Shimizu M, Kita Y, Yoshio H, Ino H, Misawa K, Matsuyama T, Mabuchi H. Quantitative evaluation of the rate of myocardial interstitial fibrosis using a personal computer. Jpn Circ J 1997; 61: 781-786.

28 Sauzeau V, Sevilla MA, Rivas-Elena JV, de Alava E, Montero MJ, Lopez-Novoa JM, Bustelo XR. Vav3 proto-oncogene deficiency leads to sympathetic hyperactivity and cardiovascular dysfunction. Nat Med 2006; 12: 841-845.

29 Weiss JL, Frederiksen JW, Weisfeldt ML. Hemodynamic determinants of the timecourse of fall in canine left ventricular pressure. J Clin Invest 1976; 58: 751-760.
30 Nakamura K, Kusano K, Nakamura Y, Kakishita M, Ohta K, Nagase S, Yamamoto M, Miyaji K, Saito H, Morita H, Emori T, Matsubara H, Toyokuni S, Ohe T. Carvedilol decreases elevated oxidative stress in human failing myocardium. Circulation 2002; 105: 2867-2871.

31 Sakata Y, Masuyama T, Yamamoto K, Doi R, Mano T, Kuzuya T, Miwa T, Takeda H, Hori M. Renin angiotensin system-dependent hypertrophy as a contributor to heart failure in hypertensive rats: different characteristics from renin angiotensin system-independent hypertrophy. J Am Coll Cardiol 2001; 37: 293-299.

32 Conrad CH, Brooks WW, Hayes JA, Sen S, Robinson KG, Bing OH. Myocardial fibrosis and stiffness with hypertrophy and heart failure in the spontaneously hypertensive rat. Circulation 1995; 91: 161-170.

33 Harada K, Sugaya T, Murakami K, Yazaki Y, Komuro I. Angiotensin II type 1A receptor knockout mice display less left ventricular remodeling and improved survival after myocardial infarction. Circulation 1999; 100: 2093-2099.

34 Fiordaliso F, Li B, Latini R, Sonnenblick EH, Anversa P, Leri A, Kajstura J. Myocyte death in streptozotocin-induced diabetes in rats in angiotensin II- dependent. $L a b$ Invest 2000; 80: 513-527.

35 Yoshida J, Yamamoto K, Mano T, Sakata Y, Nishikawa N, Nishio M, Ohtani T, Miwa T, Hori M, Masuyama T. AT1 receptor blocker added to ACE inhibitor provides benefits at advanced stage of hypertensive diastolic heart failure. Hypertension 2004; 43: 686-691.

36 Weber KT. Extracellular matrix remodeling in heart failure: a role for de novo angiotensin II generation. Circulation 1997; 96: 4065-4082.

37 Weber KT. Targeting pathological remodeling: concepts of cardioprotection and reparation. Circulation 2000; 102: 1342-1345.

38 Varo N, Etayo JC, Zalba G, Beaumont J, Iraburu MJ, Montiel C, Gil MJ, Monreal I, Diez J. Losartan inhibits the post-transcriptional synthesis of collagen type I and reverses left ventricular fibrosis in spontaneously hypertensive rats. J Hypertens 1999; 17: 107-114.

39 Tanaka Y, Gleason CE, Tran PO, Harmon JS, Robertson RP. Prevention of glucose toxicity in HIT-T15 cells and Zucker diabetic fatty rats by antioxidants. Proc Natl Acad Sci USA 1999; 96: 10857-10862.

40 Bendall JK, Cave AC, Heymes C, Gall N, Shah AM. Pivotal role of a gp91(phox)containing NADPH oxidase in angiotensin II-induced cardiac hypertrophy in mice. Circulation 2002; 105: 293-296.

41 Liu J, Yang F, Yang XP, Jankowski M, Pagano PJ. NAD(P)H oxidase mediates angiotensin II-induced vascular macrophage infiltration and medial hypertrophy. Arterioscler Thromb Vasc Biol 2003; 23: 776-782.

42 Hirotani S, Otsu K, Nishida K, Higuchi Y, Morita T, Nakayama H, Yamaguchi O, Mano T, Matsumura Y, Ueno H, Tada M, Hori M. Involvement of nuclear factor-kappaB and apoptosis signal-regulating kinase 1 in G-protein-coupled receptor agonist-induced cardiomyocyte hypertrophy. Circulation 2002; 105: 509-515.

43 Yamamoto K, Redfield MM, Nishimura RA. Analysis of left ventricular diastolic function. Heart 1996; 75: 27-35.

44 Sakata Y, Yamamoto K, Mano T, Nishikawa N, Yoshida J, Nakayama H, Otsu K, Suzuki K, Tada M, Hori M, Miwa T, Masuyama T. Angiotensin II type 1 receptor blockade prevents diastolic heart failure through modulation of $\mathrm{Ca}^{2+}$ regulatory proteins and extracellular matrix. J Hypertens 2003; 21: 1737-1745.

45 Young ME, McNulty P, Taegtmeyer H. Adaptation and maladaptation of the heart in diabetes: Part II: potential mechanisms. Circulation 2002; 105: 1861-1870.

46 Cooper ME. Pathogenesis, prevention, and treatment of diabetic nephropathy. Lancet 1998; 352: 213-219.

47 Heart Outcomes Prevention Evaluation Study Investigators. Effects of ramipril on cardiovascular and microvascular outcomes in people with diabetes mellitus: results of the HOPE study and MICRO-HOPE substudy. Lancet 2000; 355: 253-259.

48 Malhotra R, Sadoshima J, Brosius III FC, Izumo S. Mechanical stretch and angiotensin II differentially upregulate the renin-angiotensin system in cardiac myocytes in vitro. Circ Res 1999; 85: 137-146.

49 Fang ZY, Prins JB, Marwick TH. Diabetic cardiomyopathy: evidence, mechanisms, and therapeutic implications. Endocr Rev 2004; 25: 543-567.

50 Stewart RA, Joshi J, Alexander N, Nihoyannopoulos P, Oakley CM. Adjustment for the influence of age and heart rate on Doppler measurements of left ventricular filling. $\mathrm{Br}$ Heart J 1992; 68: 608-612. 